\title{
A search for deeply-bound kaonic nuclear states at J-PARC
}

S. Ajimura ${ }^{1}$, G. Beer ${ }^{2}$, H. Bhang ${ }^{3}$, M. Bragadireanu ${ }^{7,17}$, P. Buehler ${ }^{4}$, L. Busso ${ }^{5,6}$, M. Cargnelli ${ }^{4}$, S. Choi $^{3}$, C. Curceanu $^{7}$, S. Enomoto ${ }^{8}$, D. Faso ${ }^{5,6}$, H. Fujioka ${ }^{9}$, Y. Fujiwara ${ }^{10}$, T. Fukuda ${ }^{11}$, Y. Fukuda ${ }^{12}$, C. Guaraldo ${ }^{7}$, T. Hashimoto ${ }^{10}$, R. S. Hayano ${ }^{10}$, T. Hiraiwa ${ }^{9}$, M. Iio ${ }^{13}$, M. Iliescu ${ }^{7}$, K. Inoue ${ }^{8}$, T. Ishikawa ${ }^{10}$, S. Ishimoto ${ }^{14}$, T. Ishiwatari ${ }^{4}$, K. Itahashi $^{13}$, M. Iwai ${ }^{14}$, M. Iwasaki ${ }^{12,13}$, P. Kienle ${ }^{4,15}$, H. Kou ${ }^{12}$, J. Marton ${ }^{4}$, Y. Matsuda ${ }^{16}$, Y. Mizoi ${ }^{11}$, O. Morra ${ }^{5}$ T. Nagae ${ }^{9}$, H. Noumi ${ }^{1}$, H. Ohnishi ${ }^{13}$, S. Okada ${ }^{7}$, H. Outa ${ }^{13}$, D. Pietreanu ${ }^{7}$, Y. Sada ${ }^{9}$, A. Sakaguchi ${ }^{8}$, F. Sakuma ${ }^{13}$, M. Sato ${ }^{10}$, M. Sekimoto ${ }^{14}$, D. Sirghi ${ }^{7,17}$, F. Sirghi ${ }^{7,17}$, K. Suzuki ${ }^{4}$, S. Suzuki ${ }^{14}$, T. Suzuki ${ }^{10}$, H. Tatsuno ${ }^{10}$, M. Tokuda ${ }^{12}$, D. Tomono ${ }^{13}$, A. Toyoda ${ }^{14}$, K. Tsukada ${ }^{13}$, E. Widmann ${ }^{4}$, T. Yamazaki ${ }^{10,13}$, H. Yim ${ }^{3}$, and J. Zmeskal ${ }^{4}$

1 Research Center for Nuclear Physics (RCNP), Osaka University, Osaka, 567-0047, Japan

2 Department of Physics and Astronomy, University of Victoria, Victoria BC V8W 3P6, Canada

3 Department of Physics, Seoul National University, Seoul, 151-742, South Korea

4 Stefan-Meyer-Institut für subatomare Physik, A-1090 Vienna, Austria

5 INFN Sezione di Torino, Torino, Italy

6 Dipartimento di Fisica Generale, Universita' di Torino, Torino, Italy

7 Laboratori Nazionali di Frascati dell' INFN, I-00044 Frascati, Italy

8 Department of Physics, Osaka University, Osaka, 560-0043, Japan

9 Department of Physics, Kyoto University, Kyoto, 606-8502, Japan

10 Department of Physics, The University of Tokyo, Tokyo, 113-0033, Japan

11 Laboratory of Physics, Osaka Electro-Communication University, Osaka, 572-8530, Japan

12 Department of Physics, Tokyo Institute of Technology, Tokyo, 152-8551, Japan

13 RIKEN Nishina Center, RIKEN, Saitama, 351-0198, Japan

14 High Energy Accelerator Research Organization (KEK), Ibaraki, 305-0801, Japan

15 Technische Universiät München, D-85748, Garching, Germany

16 Graduate School of Arts and Sciences, The University of Tokyo, Tokyo, 153-8902, Japan

17 National Institute of Physics and Nuclear Engineering - IFIN HH, Romania

\begin{abstract}
The J-PARC E15 experiment will be performed to search for the simplest kaonic nuclear bound state, $K^{-} p p$, by the in-flight ${ }^{3} \mathrm{He}\left(K^{-}, \mathrm{n}\right)$ reaction. The exclusive measurement can be performed by a simultaneous measurement of the missing mass using the primary neutron and the invariant mass via the expected decay, $K^{-} p p \rightarrow \Lambda p \rightarrow p \pi^{-} p$. In this report, an overview of the experiment and the preparation status are presented.
\end{abstract}

\section{Introduction}

Recently, the investigation of a deeply bound kaonic nucleus have been progressed in both the theoretical and experimental sides. Theoretically, many works support the existence of deeply-bound kaonic nuclear states, but the calculated binding energies and widths are not consistent from each other. For example, the predictions of the binding energy of the $K^{-} p p$ state vary from deep $(\sim 50-130$ $\mathrm{MeV})$ down to shallow $(\sim 20 \mathrm{MeV})$, and the width from wide $(\sim 100 \mathrm{MeV})$ down to narrow $(\sim 30 \mathrm{MeV})[1-8]$. In fact, it is worth noting that several calculations for the expected spectra of $K^{-} p p$ state have been developed $[9,10]$ very recently.

On the other hand, there are several experimental reports of search for deeply-bound kaonic nuclear states by invariant mass spectroscopy by detecting decay particles, and/or missing mass spectroscopy using $\left(K^{-}, N\right)$ reactions [11-16]. However, the situation is still controversial be- cause there is no conclusive evidence for the observation of such bound states.

In order to clarify this controversial issue, the J-PARC E15 experiment was proposed to search for the simplest kaonic nuclear bound states, namely $K^{-} p p$, via the in-flight ${ }^{3} \mathrm{He}\left(K^{-}, n\right)$ reaction using $1.0 \mathrm{GeV} / \mathrm{c} K^{-}$beam [17]. This experiment has an advantage that the exclusive measurement can be performed by a simultaneous measurement of missing mass spectrum using the primary neutron and invariant mass spectroscopy via the expected decay $K^{-} p p \rightarrow$ $\Lambda p \rightarrow p \pi^{-} p$.

\section{Experimental method}

The E15 experiment will be performed at the K1.8BR beamline in the Hadron hall at the J-PARC 50-GeV proton synchrotron. The layout of the K1.8BR and the E15 spectrometer are shown in Fig. 1 The spectrometer consist of four 
parts, namely Beam-line spectrometer, Cylindrical Detector System (CDS) with liquid ${ }^{3} \mathrm{He}$ target system, Beam Sweeping Magnet and Neutron TOF wall. A secondary $\mathrm{K}^{-}$beam is focused on the liquid ${ }^{3} \mathrm{He}$ target which is located at the center of the CDS. The decay particles from the expected decay $K^{-} p p \rightarrow \Lambda p \rightarrow p \pi^{-} p$ are detected by the CDS, and the neutron counter detects forward neutrons whose flight length are about $15 \mathrm{~m}$. Incident kaons which pass through the target are bent by the sweeping magnet which is placed just after the CDS. The expected spectrometer performance for the $K^{-} p p$ measurement is 9.2 $\mathrm{MeV} / \mathrm{c}^{2}(\sigma)$ for the missing-mass resolution via neutron and $16 \mathrm{MeV} / \mathrm{c}^{2}$ for the invariant-mass resolution via the $K^{-} p p$ decay, where we assume the $K^{-} p p$ binding energy to be $100 \mathrm{MeV} / \mathrm{c}^{2}$. In the following section, the experimental components and preparation status are described.

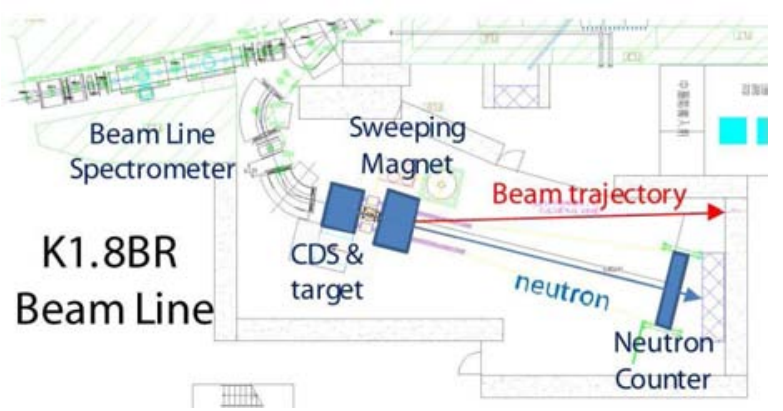

Fig. 1. Schematic view of the K1.8BR experimental hall.

\section{Preparation status of the E15 spectrometer}

\subsection{Beam-line spectrometer}

As shown in Fig.1, the secondary beam generated at the production target is momentum analyzed, mass selected and delivered to the experimental target by magnetic optical elements and an electrostatic separator. The beamline spectrometer consists of beam line magnets, tracking chambers, hodoscope, cherenkov counters and timing detectors. The momentum of the incident kaons is $1.0 \mathrm{GeV} / \mathrm{c}$ with a design intensity of $0.6 \times 10^{6}$ kaons per a spill of 3.5 second (the flat top of the spill is 0.7 second). The momentum resolution of the incident kaon with the beam line spectrometer is expected to be $0.1 \%$. Because we will perform at least two experiments at the K1.8BR beam line, namely E15 experiment and E17 experiment which is the precision measurement of X-rays from kaonic ${ }^{3} \mathrm{He}$ using stopped $K^{-}$beam [18], several setting of the beam momentum are needed, that is $1.0 \mathrm{GeV} / \mathrm{c}$ for $\mathrm{E} 15$ and $0.75 \mathrm{GeV} / \mathrm{c}$ for E17.

In February 2009, the first experiment for the beam tuning at the K1.8BR was performed using $1.1 \mathrm{GeV} / \mathrm{c}$ unseparated positive beam. As shown in Fig.2, kaons were successfully identified by the time of flight method, where the flight length was about $7.7 \mathrm{~m}$ and the calculated time difference between kaon and pion is 2.3 nsec. From the autumn 2009 , the tuning for $0.75 \mathrm{GeV} / \mathrm{c}$ beam started.

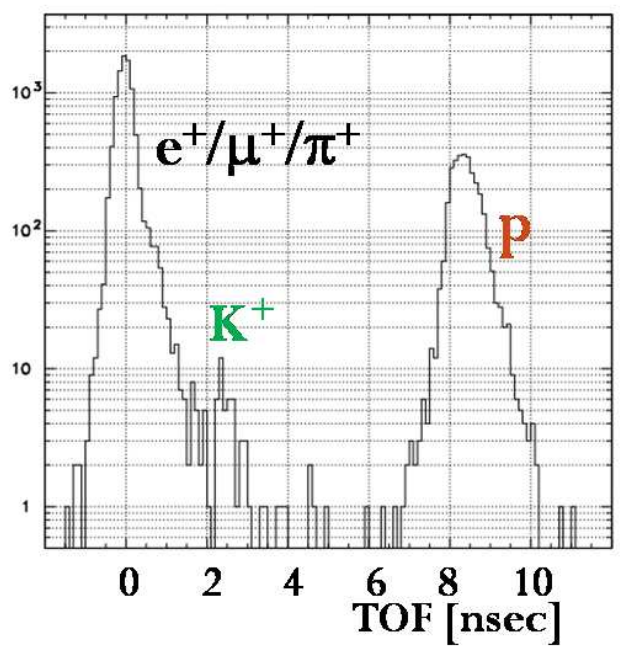

Fig. 2. The obtained TOF spectrum of the beam tune experiment at February 2009. The flight length is about $7.7 \mathrm{~m}$, and the calculated time difference between kaon and pion is $2.3 \mathrm{nsec}$.

\subsection{Cylindrical Detector System (CDS)}

A schematic view of the CDS is shown in Fig3. All detectors are configured cylindrically. Trajectory of particles is reconstructed with the Cylindrical Drift Chamber (CDC) which operates in a magnetic field of $0.5 \mathrm{~T}$ provided by the solenoid magnet. The Cylindrical Detector Hodoscope $(\mathrm{CDH})$ which surrounds the CDC is used for the trigger and the particle identification counter. The solid angle of CDS from the center is about $7.4 \mathrm{sr}$.

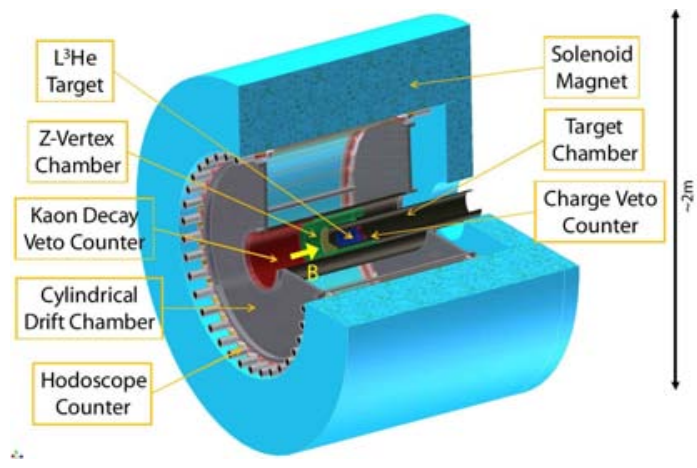

Fig. 3. Schematic view of the CDS.

\subsubsection{Solenoid magnet}

The spectrometer magnet is a solenoid type which has a bore diameter of $1.2 \mathrm{~m}$ and an overall length of $1.2 \mathrm{~m}$, with 
a weight of 23 tons. At the center of the magnet, the field strength is set to be $0.5 \mathrm{~T}(0.7 \mathrm{~T}$ at a maximum $)$. The magnet is already installed in the K1.8BR beam line, and the excitation test will be done in this fiscal year.

\subsubsection{Cylindrical Drift Chamber (CDC)}

Figure 4 shows the schematic view of CDC. The CDC consists of two aluminum end-plates of $20 \mathrm{~mm}$ thickness, a CFRP tube with $1 \mathrm{~mm}$ thick as a inner wall and six aluminum blocks which are placed outside the tracking volume. The CDC uses gold-plated tungsten of $30 \mu \mathrm{m} \phi$ for the sense wires and gold-plated aluminum of $100 \mu \mathrm{m} \phi$ for the field and guard wires. These wires are supported by feedthroughs with a bush fixing the wire position. The total length along beam axis of tracking volume is about 840 $\mathrm{mm}$. The $\operatorname{argon}(50 \%)$-ethane $(50 \%)$ mixed gas is used at 1 atm. The CDC has 15 layers of hexagonal cells with typical drift length of $9 \mathrm{~mm}$, which are grouped into 7 super layers (A1,U1,V1,A2,U2,V2,A3) as shown in Fig. 5. The information of the longitudinal position is obtained by 8 stereo layers with tilt angle of typically 3.5 degree. The number of readout channels is 1816 and the total number of wires in $\mathrm{CDC}$ is 8064 . The signal is discriminated and transfered to LVDS signal by an amplifier-shaper-discriminator (ASD) on CDC. The LVDS signal is converted to ECL signal near CDS and delivered into counting house using $50 \mathrm{~m}$ flatcable.

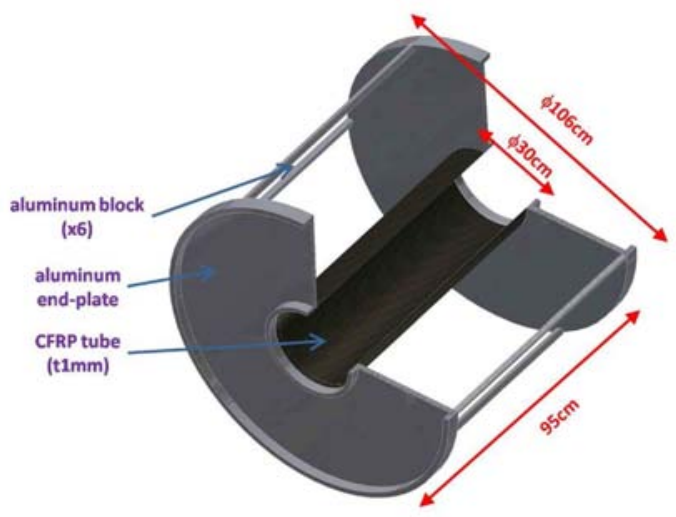

Fig. 4. Schematic view of CDC

The commissioning of CDC is going on and typical spacial resolution of $200 \mu \mathrm{m}$ is achieved by measuring the cosmic rays as shown in Fig. 6.

The CDC was installed inside the solenoid magnet in Dec. 2009 as shown in Fig. 7. The commissioning with the magnetic field will be started in this fiscal year.

\subsubsection{Cylindrical Detector Hodoscope (CDH)}

The Cylindrical Detector Hodoscope (CDH) is located at a radius of $544 \mathrm{~mm}$ from the center of CDS covering a polar angle range from 54 to 126 degrees. The $\mathrm{CDH}$ consists

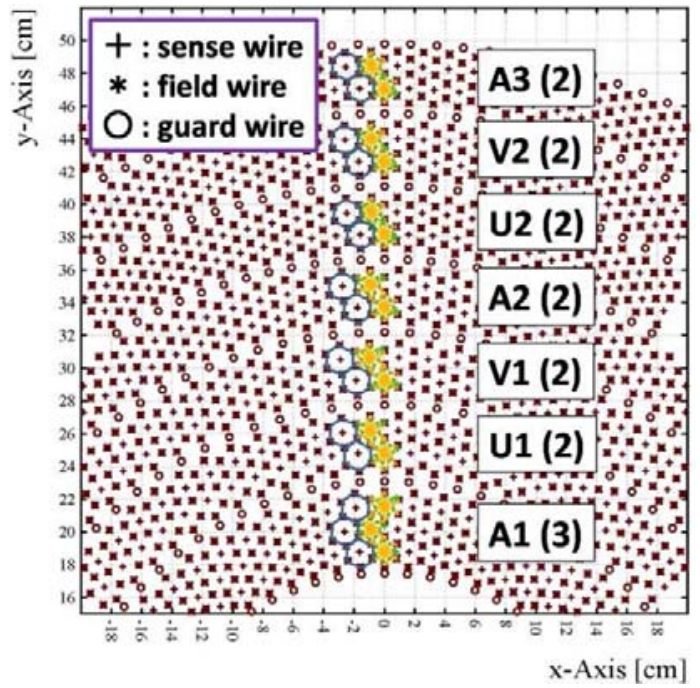

Fig. 5. Cell structure of CDC. There are 15 layers of hexagonal cells with typical drift length of $9 \mathrm{~mm}$, which are grouped into 7 super layers (A1,U1,V1,A2,U2,V2,A3).

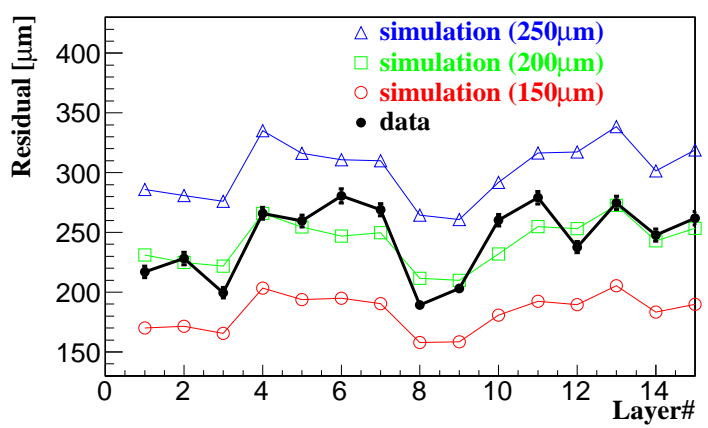

Fig. 6. Layer dependences of residuals in $\sigma$. The black circles mean the result from cosmic ray data. The open circles, squares and triangles mean the estimation by simulation assuming the intrinsic resolution of $150 \mu \mathrm{m}, 200 \mu \mathrm{m}$ and $250 \mu \mathrm{m}$, respectively.

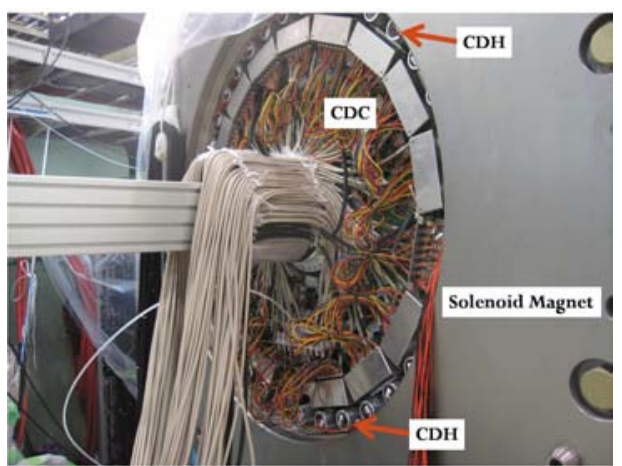

Fig. 7. A picture of CDS. CDC and $\mathrm{CDH}$ are installed in the solenoid magnet.

of 36 segments, and each segment is individually mounted on the inner wall of the solenoid magnet. The scintillators are made of Eljen EJ-560, whose dimensions are $790 \mathrm{~mm}$ in length, $99 \mathrm{~mm}$ in width and $30 \mathrm{~mm}$ in thickness. Hama- 
matsu type R7761 fine-mesh Photo Multipliers (PMT) with 1.5 inch diameter and 19 dynode stages are selected. The measured time resolution of the $\mathrm{CDH}$ with cosmic rays is typically 71 psec, achieving the design goal. All CDH segments are already installed as shown in Fig. 7.

\subsection{Liquid ${ }^{3} \mathrm{He}$ target system}

The design of the liquid ${ }^{3} \mathrm{He}$ target system is shown in Fig. 8. Basic design for the target system is based on the techniques developed for the ${ }^{4} \mathrm{He}$ target system used by KEKPS E471, E549 and E570 experiments [19]. The liquid ${ }^{4} \mathrm{He}$ is supplied from a dewar placed outside the separator but inside the target system. From separator, only liquid ${ }^{4} \mathrm{He}$ is fed into the evaporator which is placed under the separator. It is then decompressed down to 1 Torr, where the temperature is kept to be $1.3 \mathrm{~K}$. The chamber for the ${ }^{3} \mathrm{He}$ is placed under the evaporator, and is in contact with the heat exchanger. The ${ }^{3} \mathrm{He}$ gas is cooled down and liquefied in the heat exchanger, and the target cell which is almost $1 \mathrm{~m}$ away from the heat exchanger is filled with liquid ${ }^{3} \mathrm{He}$. The ${ }^{3} \mathrm{He}$ target system for E15 experiment was already completed. The details of target system are reported in [20,21].

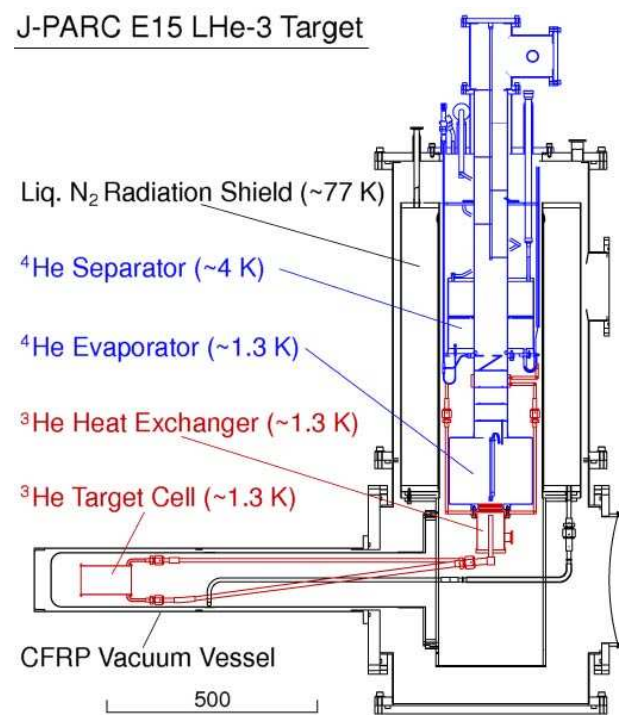

Fig. 8. Schematic view of the liquid ${ }^{3} \mathrm{He}$ target system.

\subsection{Neutron TOF wall}

The neutron counter is the same with the counter used for the KEK-PS E549 experiment. The neutron counter consists of an scintillator array covering an area of $320 \mathrm{~cm}$ in width, $150 \mathrm{~cm}$ in height and $35 \mathrm{~cm}$ in thickness. Each scintillation counter has dimensions of $20 \mathrm{~cm} \times 150 \mathrm{~cm} \times$ $5 \mathrm{~cm}$. The counters will be rearranged for the E15 experiment in 2010, using a new support frame shown in Fig. 9.

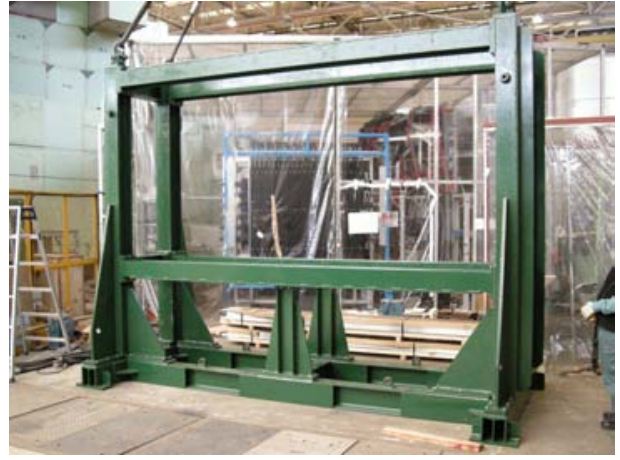

Fig. 9. Picture of the support frame of the neutron TOF wall.

\section{Conclusion}

The J-PARC E15 experiment will be performed at the $\mathrm{K} 1.8 \mathrm{BR}$ beam line at the J-PARC $50 \mathrm{GeV}$ proton synchrotron in order to clarify the present controversial situation of the existence of the kaonic nuclear bound state. This experiment is the exclusive measurement of the simplest kaonic nuclear bound state, $K^{-} p p$, by the missing mass spectroscopy via neutron detection, and the invariant mass reconstruction via the decay, $K^{-} p p \rightarrow \Lambda p \rightarrow p \pi^{-} p$. The tuning of the $1.1 \mathrm{GeV} / \mathrm{c}$ beam was performed and $K^{+}$s were successfully identified by TOF measurement in February 2009. Many of detector components were already completed, and others will be ready in 2010 . The data taking of E15 experiment will be started soon.

\section{References}

1. Y. Akaishi and T. Yamazaki, Phys. Rev. C 65, 044005 (2002)

2. T. Yamazaki and Y. Akaishi, Phys. Lett. B 535, 70 (2002)

3. A.N. Ivanov, P. Kleine, J. Marton and E. Widmann, nucl-th/0512037

4. N.V. Shevchenko, A. Gal and J. Mares, Phys. Rev. Lett. 98, 082301 (2007)

5. N.V. Shevchenko, A. Gal, J. Mares and J. Revai, Phys. Rev. C 76, 044004 (2007)

6. Y. Ikeda and T. Sato, Phys. Rev. C 76, 035203 (2007)

7. A. Dote, T. Hyodo and W. Weise, arXiv:0811.0869

8. T. Nishikawa and Y. Kondo, Phys. Rev. C 77, 055202 (2008)

9. T. Koike and T. Harada, Int. J. Mod. Phys. A 24 : 21262133 (2009)

10. J. Yamagata et al., Mod. Phys. Lett. A 23, 2528 (2008)

11. M. Sato et al., Phys. Lett. B 659, 107 (2008)

12. T. Suzuki et al., Mod. Phys. Lett. A 24, 442-445 (2009)

13. T. Kishimoto et al., Prog. Theor. Phys. 118, 181 (2007)

14. M. Agnello et al., Phys. Rev. Lett. 94, 212303 (2005)

15. G. Bendiscioli et al., Nucl. Phys.A 789, 222 (2007)

16. T. Yamazaki et al., arXiv:0810.5182 [nucl-ex]

17. M. Iwasaki et al., Proposal of J-PARC 50-GeV PS “A search for deeply-bound kaonic nuclear states by inflight ${ }^{3} \mathrm{He}\left(\mathrm{K}^{-}, \mathrm{n}\right)$ reaction", (2006) 


\section{$19^{\text {th }}$ International IUPAP Conference on Few-Body Problems in Physics}

18. R.S. Hayano et al., Proposal of J-PARC 50-GeV PS "Precision spectroscopy of Kaonic Helium $33 d \rightarrow 2 p$ X-rays", (2006)

19. M. Sato, et al., Nucl. Instr. and Meth. A 606 (2009) 233-237

20. M. Iio et al., for J-PARC E17 collaboration, contribution to this conference.

21. Y. Fujiwara et al., for J-PARC E17 collaboration, contribution to this conference. 